\title{
The Effects of Time Pressure on Temporal Overestimation Due to Threat
}

\author{
Jason Tipples $^{1, \dagger}{ }^{, *}$, Michael Lupton ${ }^{2, *}$ and David George ${ }^{2}$ \\ ${ }^{1}$ Psychology Group, Leeds Beckett University, Leeds, UK \\ ${ }^{2}$ Department of Psychology, University of Hull, Hull, UK
}

Received 17 August, 2020; accepted 18 December, 2020

† Joint first authors.

* To whom correspondence should be addressed. E-mail: W.Tipples@leedsbeckett.ac.uk 


\begin{abstract}
How does emotion change the way we perceive time? Studies have shown that we overestimate the duration of faces that express anger of fear - an effect that has been explained as due the speeding of a pacemaker that resides within an internal clock. Here, we test the idea that attending longer to facial threat leads to an overestimation of time. Seventy participants (16 male) estimated the duration of angry, fearful and neutral expressions under conditions designed to either reduce attention to time (by emphasising speedy responses) or lengthen attention to time (by emphasising accuracy). Results were modelled using Bayesian Multilevel Logistic Regression. The results replicate previous findings: speed emphasis reduced temporal sensitivity and led to both a higher overall proportion of long responses and faster reaction times. Facial threat attenuated the drop in temporal sensitivity due to speed instructions supporting the idea that people prolong attention to threat (even when they are not directly instructed to do so). We relate the findings to research into attention bias to threat and more broadly to models of perceptual decision making.
\end{abstract}




\section{Introduction}

Our perception of time changes when we feel aroused. For example, people typically overestimate the duration of faces expressing states of high arousal (e.g., Doi \& Shinohara, 2009; Fayolle \& Droit-Volet, 2014; Gil \& Droit-Volet, 2011; Gil et al., \& Droit-Volet, 2007; Tipples, 2011) and moreover, this effect is increased in individuals with high levels of anxiety and self-reported fearfulness (Bar-Haim et al., 2010; Tipples, $2008,2011)$. One explanation for this effect is that emotion speeds a pacemaker that resides within an internal clock. Here, we test the idea that people prolong attention to angry and fearful expressions, and this causes the overestimation effect. To do this we examined the effects of time pressure (Klapproth \& Müller, 2008; Klapproth \& Wearden, 2011) on time estimates for angry and fearful expressions.

Researchers have used the temporal bisection task to test whether faces expressing states of high arousal might distort our perception of time. Specifically, in a pioneering study (Droit-Volet et al., 2004) participants were asked to judge whether angry, happy, sad and neutral facial expressions were displayed for a duration that was more similar to either a standard short or a long duration that they had learnt earlier. Participants responded long more frequently following angry, happy and, to a lesser extent, sad facial expressions compared to neutral facial expressions. In other words, emotional expressions were judged to last longer; time was overestimated. The finding is both reliable - it has been replicated repeatedly - and also, a valid indicator of emotional reactivity - it is associated with individual differences in emotion (including anxiety) and occurs in response to a variety of emotion-evoking stimuli. A key question, however, is how emotional stimuli affect time. Two explanations have been given pacemaker speeding (described next) and attention.

One explanation for the effects of facial expressions on time perception is that emotional arousal increases the rate of a pacemaker mechanism that resides within an internal clock. Internal-clock models of timing (Gibbon et al., 1984; Rammsayer \& Ulrich, 2001; Treisman, 1963; Treisman et al., 1990; Zakay \& Block, 1997) typically include: (1) a pacemaker that emits units of time (or pulses) at specific rate, (2) an attention-controlled switch that controls the flow of pulses, and (3) a counter where 
perceived time is calculated based on the total number of counted units. One variant of this basic model includes an attention-controlled gate (Block \& Zakay, 1996; Zakay \& Block, 1995) that precedes the switch mechanism - the gate opens wider when more attention is allocated to time. One prediction of the internal-clock model is that the effects of pacemaker speeding will multiply as duration increases. In the temporal bisection task, a multiplicative pattern for emotion would be recorded if emotion increased the gradient of the psychophysical slope for duration. The multiplicative nature of pacemaker speeding has been compared to the additive effects that are thought to reflect the closure of the switch mechanism. In the internal-clock model, the switch closes when timing starts to allow units of time to enter the counter. The switch reopens when timing ends. Delays in the closure and reopening of the switch are expected to be additive with increases in time because they occur in an 'all-or-none' manner leading to the addition (or subtraction) of a fixed number of units of time. In the temporal bisection task, an additive pattern for emotion is indexed by a shift in the intercept of the psychophysical slope for duration. If emotion delays the closure of the switch then timing will start later and time will be underestimated - a rightward shift in the psychophysical function for emotion. If emotion delays the reopening of the switch, then timing will end later, and time will be overestimated leading to a rightward shift in the psychophysical function for emotion.

Emotional stimuli both increase arousal and lead to overestimation and therefore, the effects have been attributed the effects of emotion on time to pacemaker speeding due to arousal. A pattern of underestimation - suggestive of a delay in opening of the switch - has been reported for emotionally arousing images (Lui et al., 2011). However, in the latter study emotional stimuli were irrelevant distractors interleaved between two timing stimuli - a stimulus context that may have created attentional competition. Most studies have presented the emotion stimuli as the to-be-timed stimuli and have reported an overestimation rather than underestimation of time.

Although overestimation rather than underestimation is typically reported for emotion stimuli, a multiplicative overestimation pattern - expected for pacemaker speeding - has not been reported consistently across studies. For angry facial expressions specifically, some studies have reported a multiplicative pattern 
(Droit-Volet et al., 2004) whereas other studies have recorded an additive effect (e.g., Fayolle \& Droit-Volet, 2014). An additive overestimation effect supports the idea of a slower reopening of the switch mechanism and therefore, evidence is equivocal with respect to the pacemaker-speeding account.

Isolating arousal-based, pacemaker-speeding effects from attentional effects is made difficult for several reasons. First, an initial fast arousal effect might give way to attentional effects. Second, there is more than one variety of attention that needs to be considered. Specifically, sustained rather than the initial orienting of attention might mediate the effects of emotion on timing. As noted recently (Lake et al., 2016) a general assumption of pacemaker accumulator models is that full attention is given to the stimulus under normal conditions and consequently attention can only be distracted from timing. This assumption may hold for highly motivated laboratory rats on which the internal-clock models was based but seems less likely for research participants who wish to leave the laboratory quickly. An individual who fails to sustain attention to the full stimulus duration will have a shallower psychophysical slope for duration compared to an individual who always attends to the full duration (Matthews \& Meck, 2016). However, when participants are motivated to look at an image - a person staring at them in a threatening manner for example - it makes sense to keep attention tightly fixed on the person for as long as possible and not let attention waiver during stimulus presentation. In short, attending to an image for longer because it has motivational relevance (e.g., a picture of spider to a spider-phobic or a fearful face to a participant who is generally anxious) is one way in which attention might mediate the effects of emotion on time estimates.

A manipulation that either prolongs or shortens attention to the longest durations is one way of testing the prolonged-attention account for an overestimation effect for facial expressions and other emotion stimuli. If participants attend longer to emotion stimuli compared to neutral stimuli then the prediction is that emotion will attenuate or lessen any manipulation designed to reduce attention to time. One manipulation that is thought to reduce attention to the longest durations is time pressure. The effects of time pressure on time perception were originally studied by Klapproth and colleagues (Klapproth \& Müller, 2008; Klapproth \& Wearden, 2011) using the temporal generalisation task. In the temporal generalisation task, participants 
judge whether a comparison duration is either the same as or different from a standard duration. Plotting the proportion of same responses against the comparison duration reveals a temporal gradient. Klapproth and colleagues found that time pressure shifted the temporal generalisation gradient leftwards - overall participants responded 'same' more frequently irrespective of the actual duration of the comparison stimulus. Less consistently, time pressure also reduced the temporal gradient itself. Klapproth and colleagues' interpretation of their findings was that under time pressure participants shortened their timing of the longest durations and consequently, the average experience of time was 'truncated' under time pressure compared to accuracy emphasis. Building on the research of Klapproth and colleagues, our hypothesis is that if participants prolong attention to emotion stimuli for the longest durations then this will attenuate the effect of time pressure.

\subsection{This Study}

To provide some consistency with previous studies of the effects of emotion on time estimates we used the temporal bisection task rather than the temporal generalisation task used by Klapproth and colleagues. A between-subjects design was used participants were randomly assigned to either time pressure or accuracy conditions. In the accuracy condition participants were instructed to be as accurate as possible. In the time pressure condition, responding quickly was emphasised and feedback was given as "too slow" if responses were slower than $500 \mathrm{~ms}$. There were several predictions. First, compared to the accuracy condition participants in the time pressure will respond faster and show a flattening of the psychophysical slope for duration. A flattening of the psychophysical slope due to time pressure is expected because this is the pattern that is also found in studies of magnitude estimation more generally (see for example; Ratcliff, 2014). The central prediction is that the flattening of the psychophysical slope for participants under time pressure will be smaller in magnitude for threat-related (angry and fearful) expressions compared to neutral expressions. 


\section{Methods}

\subsection{Participants}

Seventy undergraduate psychology students were randomly assigned to either the speed condition (mean age $=21,7$ males and 28 females) or the accuracy condition (mean age $=23,9$ males and 26 females). The mean self-reported trait fearfulness scores did not differ between participants in the accuracy condition $(M=10.05 ; \mathrm{SD}=1.97)$ and participants in the speed condition $(\mathrm{M}=8.85 ; \mathrm{SD}=1.97), F_{1,68}=1.47, p=0.22$, $\eta_{\mathrm{p}}{ }^{2}=0.02$.

\subsection{Materials and Apparatus}

The face stimuli were the same as those used in a previous study (Tipples, 2011) that recorded an overestimation effect for angry and fearful expressions compared to a neutral face that grew in magnitude for individuals with high self-reported levels of anxiety and fearfulness. The stimuli were created using software from a commercial company called Poser 5.o (Curious Labs Inc., Santa Cruz, CA, USA). In previous research the angry and fearful faces were rated as more arousing and less pleasant than the neutral face.

\subsection{Procedure}

All participants completed learning and test phases. In the learning phase, participants were trained to discriminate short (400 $\mathrm{ms}$ ) from long $(1,600 \mathrm{~ms})$ stimulus durations. On the first eight trials, a pink oval appeared for either a short or long duration in a fixed sequence (e.g., long-short-long-short, etc.). Participants were told to expect this sequence and to press either the $\mathrm{Z}$ or $\mathrm{M}$ button on a computer keyboard to indicate whether the oval appeared for either a short or a long duration. The response mapping (e.g., $\mathrm{Z}$ for short durations and $\mathrm{M}$ for long durations) was counterbalanced across participants. Following a response, participants were presented with visual feedback lasting $500 \mathrm{~ms}$, for both correct ('yes') and incorrect ('no') decisions. The feedback was followed by a fixed $1000 \mathrm{~ms}$ inter-trial interval. In the final stage of the learning phase, the pink oval was presented for a further eight trials in a new random order for each participant. Participants continued to indicate whether the oval appeared for either short or long stimulus durations and received feedback.

During the test phase, the oval was replaced by the face stimuli. Participants were asked to (a) look at the face and (b) indicate whether the face appeared for a duration that was closer to either the short or long durations that they had learnt earlier. In the test phase, there were 21 possible trial types 
that were derived from the factorial combination of duration (400, 800, 1000, 1200, 1400 600, 1600) $\times$ expression (angry, fearful, neutral). Participants in the speed were instructed: "Please respond as quickly as possible. Accuracy is not crucial" and given the feedback "Reaction time too slow" if their reaction times were longer than $500 \mathrm{~ms}$. Participants in the accuracy group were instructed: "Please respond as accurately as possible. Speed is not important". We wanted to make all other aspects of the task as similar as possible to that used in previous research and therefore in the test phase feedback was not given for accuracy. There were eight repetitions of the 21 trial types leading to the creation of 168 trials. A new randomised trial order was created for each participant. Finally, after the main test phase participants completed the EAS Temperament Survey for Adults (EAS; Buss \& Plomin, 1984).

\section{Results}

\subsection{Reaction Times}

An initial manipulation check was conducted to establish whether participants in the speed condition responded more quickly than those in the accuracy condition. Specifically, the mean reaction times (RTs) were analyses in an expression (neutral, angry, fearful) $\times$ duration $(400,600,800,1000,1200,1400,1600) \times$ condition (speed, accuracy) mixed ANOVA with condition as the between-subjects variable. The ANOVA revealed main effects for condition, $F_{1,68}=182, p<0.001, \eta_{\mathrm{p}}{ }^{2}=0.73$ and duration, $F_{1,68}=$ $5.50, p=0.022, \eta_{\mathrm{p}}{ }^{2}=0.07$ and an interaction between condition and duration, $F_{1,68}=$ 9.78, $p=0.003, \eta_{\mathrm{p}}{ }^{2}=0.13$. All other effects failed to reach the convention for statistical significance (alpha $=0.05$ ). Figure 1 shows the mean RTs in seconds as a function of duration and condition. The interaction shows that: (1) RTs were faster in speed condition and (2) participants were slowest responding as the decision became harder as the duration approaches the mean (1000 $\mathrm{ms}$ ) of the durations. For the participants in the speed condition the effect was linear - RTs became faster as the durations lengthened.

\subsection{Hierarchical Bayesian Logistic Regression}

Following Tipples (2019), the 'short' and 'long' responses for each trial were modelled in 
a multilevel Bayesian logistic regression. Details of model selection and further aspects of the modelling procedure can be found in the Supplementary appendix. To permit comparison with previous research that has not used the Bayesian approach we conducted supplementary frequentist analyses. These analyses can also be found in the Supplementary appendix. Figure 2 displays the mean proportion of long responses for each combination of expression (neutral, angry, fearful), duration and condition (speed, accuracy).

In the Bayesian framework statistical inference can be made directly on the posterior distribution of the regression model coefficients. The coefficients can be used to calculate indices such as the Weber ratio (WR) and the Bisection Point (BP). The BP refers to point of subjective equality (0.5 point on the psychometric function). The WR is an index of temporal sensitivity and is calculated by dividing the difference limen (half of the difference between the stimulus durations giving rise to $75 \%$ long responses and $25 \%$ long responses) by the BP (see also; Tipples, 2019). Relatively lower WR values indicate a greater sensitivity to time. The WR and BP have been repeatedly used in previous research on this topic and therefore, we calculated these indices from the posterior distribution of the fixed-effect regression coefficients.

Boxplots for the WRs and BPs are plotted as a function of expression and condition in Figures 3 (WRs) and 4 (BPs). Figure 3 shows that there was a marked reduction in temporal sensitivity for participants in the speed compared to the accuracy condition. However, as can be seen in Fig. 3, the key result is that in the speed but not the accuracy condition WRs were lower (indicating greater temporal sensitivity) for angry and fearful faces compared to neutral faces. The regression coefficients support this observation. Specifically, the $95 \%$ credible values for the three-way expression (fear vs neutral) $\times$ duration $\times$ condition (speed vs accuracy) interaction contrast did not contain the value zero $[\beta=0.0 .0013 ; 95 \%$ credibility interval $(\mathrm{CrI})=0.0003-0.0023]$. The three-way interaction contrast for angry expressions did contain zero, $(\beta=0.0008$; 95\% CrI $=-0.0002-0.0018)$. However, it would be a mistake to argue for the null ('zero effect;') in this condition. Instead, we express greater uncertainty over the non-null value of this effect - we are $80 \%$ rather than $95 \%$ certain that the effect does not contain zero $(80 \% \mathrm{CrI}=0.0001-0.001)$. 
Figure 4 shows that the probable BP values in the accuracy condition (left panel) were closer to mean duration (1000 ms) than those in the speed condition. In other words, instructions to be accurate improved accuracy. Also, in the accuracy condition the BPs were shifted to the left for angry and fearful faces compared to neutral faces. However, the effect was relatively small ( $<35$ milliseconds) for both comparisons. For the contrast fearful-neutral, $80 \%$ of the probability mass did not contain the value zero whereas for the contrast angry-neutral $95 \%$ did not contain zero. The same contrasts for the speed condition are difficult to interpret because shifts in the BP are not independent from shifts in the WR and, as the above analysis shows, temporal sensitivity was higher (WRs were lower) for angry and fearful expression compared to the neutral expression condition in the speed condition. Finally, in keeping with the idea that time pressure truncates temporal experience by reducing attention to the full duration (Klapproth \& Müller, 2008; Klapproth \& Wearden, 2011) the median BP values in the speed condition are all below $1000 \mathrm{~ms}$ and moreover, the BPs in the speed condition are shifted left relative to the accuracy condition. This reflects relative overestimation (the psychometric function is shifted upward on the $Y$-axis) and would be expected if (1) the average experience duration was relatively shorter in the speed condition and (2) this mean was used as comparison duration that a specific duration must pass to be categorised as long.

\section{Discussion}

The results offer support for the prolonged-attention hypothesis that people attend longer to threat-related expressions and consequently this leads them to overestimate time. When participants were instructed to respond quickly, fearful and angry expressions increased temporal sensitivity as indicated by a steepening of the psychophysical slope for duration. This finding is consistent with the idea that people spent longer attending to angry and fearful faces compared to neutral faces and consequently they responded long more often as the duration lengthened. For participants given instructions to be as accurate as possible, there was a leftward shift in the BP for angry and fearful expressions compared to neutral expressions. However, this 
overestimation effect was small in magnitude and moreover, increased temporal sensitivity due to facial threat was absent. Our interpretation of the pattern of results in the accuracy condition is that increased attention to neutral stimuli led to a reduction in the usually observed overestimation effect for threat-related expressions. Put differently, we think that the usually observed overestimation effect for emotion depends on participants not fully paying attention to neutral stimuli. The fact that a small effect of facial threat remained is most likely due to the weak manipulation of accuracy we used - participants were not given feedback but rather accuracy was stressed via verbal instructions. In sum, our results support a central role for attention in mediating the effects of facial threat on time estimates.

How can the proposed attention effects be explained in terms of the putative mechanisms of the internal-clock model? Within internal-clock models, it is possible to distinguish between the role of selective attention in starting and ending the timing process and sustained attentional effects that operate throughout the duration of the stimulus (Fernandes \& Garcia-Marques, 2020; Matthews \& Meck, 2016; Ogden et al., in press). In the internal-clock model selective attention is needed at the beginning of the stimulus to close the switch and once at the end of the timing processes to reopen the switch. Such effects are thought to produce additive ('intercept') effects on timing, namely left or rightward shifts of the bisection curve. Sustained attention can be conceptualised as affecting the clock operation in two ways. First, sustained attention might be necessary to keep the switch closed and prevent lost pulses via an opening and closing or 'flickering' (Lejeune, 1998) of the switch. Second, within the Attentional Gate Model (AGM; Block \& Zakay, 1996; Zakay, 1989) sustained attention can be conceptualised as controlling the width of an additional gate mechanism that precedes the switch. In the AGM, the gate controls the effective flow of the pulses from the pacemaker to the accumulator and therefore, when the gate is narrower fewer pulses accumulate as time progresses. In either case, a stimulus that increases sustained attention such as a threatening stimulus will lead to a reduction in lost pulses across time and an increase in the gradient of the psychophysical curve reported both here and separate research (Tipples, 2019).

Although an attentional account has been proposed to account for the effects reported here it is unlikely that such effects operate independently from arousal. Indeed, 
sustained attention is sometimes used synonymously (within attentional models) to refer to increased alertness due to arousal. According to one well-known model of attention (Petersen \& Posner, 2012) alertness can be differentiated from focused or selective attention in that it is (1) not spatially selective - it operates across the visual field - and (2) is dependent on the locus coeruleus-norepinephrine (LC-NE) system rather than the more localised brain systems for orienting attention and executive control (see Fig. 2; Petersen \& Posner, 2012).

The results also replicate and extend previous reports (Klapproth \& Müller, 2008; Klapproth \& Wearden, 2011) by showing that the effect of time pressure (speed instructions) on time estimates generalises to the temporal bisection task. Specifically, in keeping with the idea that time pressure truncates temporal experience by reducing attention to the full duration (Klapproth \& Müller, 2008; Klapproth \& Wearden, 2011) the median BP values in the speed condition were all below $1000 \mathrm{~ms}$ and moreover, the $\mathrm{BP}$ in the speed condition was shifted left relative to the accuracy condition. This reflects relative overestimation (the psychometric function is shifted upward on the $Y$-axis) and would be expected if (1) the average experience duration was relatively shorter in the speed condition and (2) the mean duration used as a comparison duration was represented by a lower value. In the accuracy condition, however, the median BP values clustered around the mean duration (1000 ms) as would be expected if participants attempted to perceive time accurately by attending to the full duration of the faces.

More broadly, the flattened psychophysical slope for duration (due to time pressure) is consistent with findings from perceptual decision-making research into the speed-accuracy trade-off. For example, in one task (Experiment 10; Ratcliff, 2014) participants were asked to decide whether the distance between two dots was large or small. Instructions to respond quickly reduced the gradient of the psychophysical function - participants were relatively more indiscriminate as they rushed to respond in time. Here, we found an analogous effect - compared to instructions to be accurate, the psychophysical function reduced in gradient. Our interpretation is similar too collecting more evidence before making a decision (by attending longer to the stimulus) leads to slower but more precise decisions.

Speed-accuracy trade-offs in perceptual decision making are frequently modelled using the sequential sampling models of decision making. Studies that have used the 
drift diffusion model (DDM; Ratcliff, 1978; Ratcliff \& McKoon, 2008) to model the effect of time pressure on perceptual decision making have shown that speed instructions lead to reduction in the boundary separation parameter - the model parameter that governs the amount of information required for a decision. Large boundary separation values indicate conservative response criteria (more information is required for a decision) whereas smaller values indicate more liberal and perhaps impulsive response criteria (less information is required for a decision). This boundary separation parameter was not modelled in previous research (Tipples, 2015) that used the temporal bisection task to study the effect of emotion on temporal decision making. It was not modelled because modelling this parameter is difficult using this task specifically (Balcı \& Simen, 2014). However, based on the current research, modelling this parameter might yet provide insight into the basic process that underlies the effect of emotion on time. The prediction for boundary separation is that emotion will increase the boundary separation parameter as participants collect more data before making a decision.

The specific roles for attention and arousal are by no means settled by the current work. Instead, our work shows that increased time pressure might be necessary for revealing attentional processes. Future work will need to consider how the effects reported here change across different duration ranges, tasks, and stimuli. Comparing duration ranges will be insightful for two reasons. First, past work indicates that attentional and arousal processes might have different time courses. For example, one study (Gil \& Droit-Volet, 2012) found that the arousal-based effect produced by the perception of emotional pictures was short-lived $(<1 \mathrm{~s})$. Second, replicating across duration ranges permits testing for a multiplicative pattern - the expected pattern for pacemaker speeding due to arousal. Finally, using different tasks (e.g., verbal estimation) will be useful because the temporal bisection task might not be sensitive to the subtle interplay between attention and arousal across time.

In summary, the current findings support the hypothesis that participants attend longer to threat-related expressions and this leads to an overestimation effect. This is consistent with the effects of emotion on timing reported in previous research (Lui et al., 2011) and more broadly consistent with the idea that people prolong attention to facial threat (Fox et al., 2002; Georgiou et al., 2005). In short, the results facilitate the 
understanding of this effect in relation to effects recorded outside the time perception literature and more specifically, shed light on our understanding of the effects of facial threat on time perception. 


\section{References}

Balcı, F., \& Simen, P. (2014). Decision processes in temporal discrimination. Acta Psychol., 149, 157-168. https://doi.org/10.1016/j.actpsy.2014.03.005

Bar-Haim, Y., Kerem, A., Lamy, D., \& Zakay, D. (2010). When time slows down: The influence of threat on time perception in anxiety. Cogn. Emot., 24, 255-263. https://doi.org/10.1080/02699930903387603

Block, R. A., \& Zakay, D. (1996). Models of psychological time revisited. In H. Helfrich (Ed.), Time and mind (pp. 171-195). Kirkland, WA, USA: Hogrefe \& Huber.

Buss, A. H., \& Plomin, R. (1984). Temperament: early developing personality traits. Hillsdale, NJ, USA: Lawrence Erlbaum Associates.

Doi, H., \& Shinohara, K. (2009). The perceived duration of emotional face is influenced by the gaze direction. Neurosci. Lett., 457, 97-100. https://doi.org/10.1016/j.neulet.2009.04.004

Droit-Volet, S., Brunot, S., \& Niedenthal, P. (2004). Perception of the duration of emotional events. Cogn. Emot., 18, 849-858. https://doi.org/10.1080/02699930341000194

Fayolle, S. L., \& Droit-Volet, S. (2014). Time perception and dynamics of facial expressions of emotions. PLoS ONE, 9, e97944. https://doi.org/10.1371/journal.pone.o097944

Fernandes, A. C., \& Garcia-Marques, T. (2020). A meta-analytical review of the familiarity temporal effect: testing assumptions of the attentional and the fluency-attributional accounts. Psychol. Bull., 146, 187-217. https://doi.org/10.1037/buloooo222

Fox, E., Russo, R., \& Dutton, K. (2002). Attentional bias for threat: evidence for delayed disengagement from emotional faces. Cogn. Emot., 16, 355-379.

Georgiou, G., Bleakley, C., Hayward, J., Russo, R., Dutton, K., Eltiti, S., \& Fox, E. (2005). Focusing on fear: Attentional disengagement from emotional faces. Vis. Cogn., 12, 145-158. https://doi.org/10.1080/13506280444000076

Gibbon, J., Church, R. M., \& Meck, W. H. (1984). Scalar timing in memory. Ann. N. Y. Acad. Sci., 423, $52-77$.

Gil, S., \& Droit-Volet, S. (2011). "Time flies in the presence of angry faces"... depending on the temporal task used! Acta Psychol., 136, 354-362. https://doi.org/10.1016/j.actpsy.2010.12.010

Gil, S., \& Droit-Volet, S. (2012). Emotional time distortions: The fundamental role of arousal. Cogn. Emot., 26, 847-862. https://doi.org/10.1080/02699931.2011.625401

Gil, S., Niedenthal, P. M., \& Droit-Volet, S. (2007). Anger and time perception in children. Emotion, 7 , 219-225. https://doi.org/10.1037/1528-3542.7.1.219

Klapproth, F., \& Müller, M. (2008). Temporal generalization under time pressure in humans. Q. J. Exp. Psychol., 61, 588-600. https://doi.org/10.1080/17470210701255572 
Klapproth, F., \& Wearden, J. H. (2011). Why do temporal generalization gradients change when people make decisions as quickly as possible? Q. J. Exp. Psychol., 64, 1646-1664. https://doi.org/10.1080/17470218.2011.564290

Lake, J. I., LaBar, K. S., \& Meck, W. H. (2016). Emotional modulation of interval timing and time perception. Neurosci. Biobehav. Rev., 64, 403-420. https://doi.org/10.1016/j.neubiorev.2016.03.003

Lejeune, H. (1998). Switching or gating? The attentional challenge in cognitive models of psychological time. Behav. Proc., 44, 127-145. https://doi.org/10.1016/so376-6357(98)ooo45-x

Lui, M. A., Penney, T. B., \& Schirmer, A. (2011). Emotion effects on timing: attention versus pacemaker Accounts. PLOS ONE 6, e21829. https://doi.org/10.1371/journal.pone.0021829

Matthews, W. J., \& Meck, W. H. (2016). Temporal cognition: Connecting subjective time to perception, attention, and memory. Psychol. Bull., 142, 865-907. https://doi.org/10.1037/buloooo045

Ogden, R., Turner, F., \& Pawling, R. (in press). An absence of a relationship between overt attention and emotional distortions to time: An eye-movement study. Timing Time Percept.

Petersen, S. E., \& Posner, M. I. (2012). The attention system of the human brain: 20 years after. Annu. Rev. Neurosci., 35, 73-89. https://doi.org/10.1146/annurev-neuro-062111-150525

Rammsayer, T., \& Ulrich, R. (2001). Counting models of temporal discrimination. Psychon. Bull. Rev., 8, 270-277. https://doi.org/10.3758/BFo3196161

Ratcliff, R. (1978). A theory of memory retrieval. Psychol. Rev., 85, 59-108. https://doi.org/10.1037/0033-295X.85.2.59

Ratcliff, R. (2014). Measuring psychometric functions with the diffusion model. J. Exp. Psychol. Hum. Percept. Perform., 40, 870-888. https://doi.org/10.1037/a0034954

Ratcliff, R., \& McKoon, G. (2008). The diffusion decision model: theory and data for two-choice decision tasks. Neural Comput., 20, 873-922. https://doi.org/10.1162/neco.2008.12-06-420

Tipples, J. (2008). Negative emotionality influences the effects of emotion on time perception. Emotion, 8, 127-131. https://doi.org/10.1037/1528-3542.8.1.127

Tipples, J. (2011). When time stands still: Fear-specific modulation of temporal bias due to threat. Emotion, 11, 74-80. https://doi.org/10.1037/aoo22015.

Tipples, J. (2015). Rapid temporal accumulation in spider fear: Evidence from hierarchical drift diffusion modelling. Emotion, 15, 742-750. https://doi.org/10.1037/emoooooo79

Tipples, J. (2019). Increased temporal sensitivity for threat: A Bayesian generalized linear mixed modeling approach. Atten. Percept. Psychophys., 81, 707-715. https://doi.org/10.3758/s13414-018-01637-9

Treisman, M. (1963). Temporal discrimination and the indifference interval. Implications for a model of the "internal clock". Psychol. Monogr. Gen. Appl., 77, 1-31. https://doi.org/10.1037/hoo93864

Treisman, M., Faulkner, A., Naish, P. L., \& Brogan, D. (1990). The internal clock: evidence for a temporal oscillator underlying time perception with some estimates of its characteristic frequency. Perception, 19, 705-743. 
Zakay, D. (1989). Subjective time and attentional resource allocation: An integrated model of time estimation. In I. Levin, \& D. Zakay (Eds), Advances in Psychology, Vol. 59 (pp. 365-397). Amsterdam, The Netherlands: Elsevier. https://doi.org/10.1016/So166-4115(08)61047-X

Zakay, D., \& Block, R. A. (1995). An attentional-gate model of prospective time estimation. In M. Richelle, V. D. Keyser, G. D. Ydeualle, \& A. Vandierendonck (Eds), Time and the dynamic control of behavior (pp. 167-178). Liege, Belgium: University of Liege Press.

Zakay, D., \& Block, R. (1997). Temporal cognition. Curr. Dir. Psychol. Sci., 6, 12-16. https://doi.org/10.1111/1467-8721.ep11512604 


\section{Figure Captions}

Figure 1. Mean reaction times (RTs) in seconds as a function of duration and condition. Error bars are bootstrapped 95\% CIs.

Figure 2. The mean proportion of long responses for each combination of expression (neutral, angry, fearful), duration and condition (speed, accuracy). The points are the mean bisection values from the fitted model.

Figure 3. Boxplot of Weber ratios as a function of expression and condition. The boxplots were created from the posterior distribution of the fixed effect regression coefficients.

Figure 4. Boxplot of bisection points (in milliseconds) as a function of expression and condition. The boxplots were created from the posterior distribution of the fixed effect regression coefficients. 


\section{Timing \& Time Perception \\ The Effects of Time Pressure on Temporal \\ Overestimation Due to Threat}

Jason Tipples, Michael Lupton and David George

Supplementary Material

Frequentist Analyses

To permit comparison with previous research that has not used the Bayesian approach we also conducted frequentist (non-Bayesian) analyses. Specifically, we estimated a psychometric curve for each person for each expression and condition, by modelling the number of long responses using a binomial generalized linear model (GLM) with a logistic link function in $R$ ( $R$ Core Team, 2018. R: A Language and Environment for Statistical Computing. R Foundation for Statistical Computing, Vienna, Austria. https://cran.r-project.org). Then, the bisection point (BP) and Weber Ratio (WR) were calculated in the same way (described in the text) used to calculate these indices for the Bayesian Model. Both the BPs and WRs were subjected to a mixed ANOVA with expression (angry, fearful, neutral) as the within-subjects variable and condition (accuracy, speed) as the between-subjects variable. The Greenhouse-Geisser correction for sphericity was applied to the degrees of freedom. For the analyses of BPs there was a clear main effect of condition $\left(F_{1,68}=182, p<0.001\right)$, reflecting lower BPs for the speed $(M=877)$ compared to the accuracy condition $(M=1027)$. There was also a main effect of expression $\left(F_{1.92,130.69}=4.73, p=0.01\right)$, reflecting lower bisection points for angry $(M=932)$ compared to the neutral expression $\left(M=973 ; t_{69}=3.53, p=0.0009\right)$. The contrast between neutral and fearful expressions $(M=951)$ was not significant $\left(t_{69}=1.61, p=0.11\right)$. The expression $\times$ condition interaction was also not significant for BPs $\left(F_{1.92,130.69}=0.65, p=0.51\right)$. For the analyses of WRs, the expression $\times$ condition interaction was significant $\left(F_{1.79,121.45}=3.65, p=0.03\right)$. There was a simple main effect of expression in the speed condition $\left(F_{1.61,54.84}=5.75, p=0.009\right)$ but not the accuracy condition, $F_{1.82,61.85}=0.31, p=0.71$. In the speed condition, WRs were lower (indicating higher temporal sensitivity) for fearful $(M=0.18)$ compared neutral expressions $(M=$ $\left.0.23 ; t_{34}=2.12, p<0.05\right)$. WRs were also lower for angry $(M=0.19)$ compared to neutral 
expressions although the effect approached rather than reached significance $\left(t_{34}=1.84, p=\right.$ 0.07). Overall, the pattern of results matches the Bayesian analyses. 